\title{
Tangence
}

\section{Phénoménologie de l'acte de lecture : l'exemple de Volkswagen Blues de Jacques Poulin}

\section{Blanca Navarro Pardiñas}

Numéro 36, mai 1992

La lecture littéraire

URI : https://id.erudit.org/iderudit/025711ar

DOI : https://doi.org/10.7202/025711ar

Aller au sommaire du numéro

Éditeur(s)

Tangence

ISSN

0226-9554 (imprimé)

1710-0305 (numérique)

Découvrir la revue

Citer cet article

Pardiñas, B. N. (1992). Phénoménologie de l'acte de lecture : l'exemple de

Volkswagen Blues de Jacques Poulin. Tangence, (36), 52-62.

https://doi.org/10.7202/025711ar d'utilisation que vous pouvez consulter en ligne.

https://apropos.erudit.org/fr/usagers/politique-dutilisation/ 


\section{Phénoménologie de l'acte de lecture: l'exemple de Volkswagen Blues de Jacques Poulin}

\section{Blanca Navarro Pardiñas}

Depuis la fin des années 1960, la figure du lecteur, longtemps oubliée des critiques, est devenue la cible de nombreuses analyses qui ont tâché, avec plus ou moins de succès, d'éclairer son activité ${ }^{1}$. Avec le temps, l'acte de lecture s'est érigé en véritable objet de recherche. Mais, l'entreprise est loin d'être terminée, la lecture étant l'un des actes les plus abstraits qui existent. Il reste encore du chemin à parcourir, voilà de quoi " faire de beaux rêves "...

Si la majorité des critiques s'accordent pour affirmer l'importance du rôle du lecteur dans l'interprétation de l'œuvre littéraire, les divergences refont surface quand vient le temps de définir précisément ses contours. Au stade où nous en sommes, il est important de multiplier les analyses concrètes de la lecture, et cela par tous les moyens possibles. Certains laboratoires de recherche s'occupent des problèmes physiologiques de la lecture. Mais cette dimension de la lecture n'est pas la nôtre. Pour nous, littéraires, il s'agit de questionner nos propres lectures, nos théories, sur des œuvres qui s'y prêtent de façon particulière. À cet égard, celles du Québécois Jacques Poulin, avec ses nombreux personnages lecteurs, nous offrent un champ de recherche privilégié. Nous nous proposons ici de travailler ce corpus, de reconstruire en fait un acte de lecture de Volkswagen Blues. Nous prendrons comme point de départ la théorie de Wolfgang Iser, de façon à explorer l'interaction entre le texte et le lecteur réel.

D'après Iser, la lecture littéraire est construite comme une interaction entre le texte et le lecteur. Ce dialogue se produit à partir de stratégies textuelles, d'éléments d'organisation du texte (personnages, dialogues, etc.). Ce sont ces stratégies qui attirent l'attention du lecteur sur des éléments de répertoire textuel renvoyant au

1 La publication de Pour une estbétique de la réception de Hans Robert Jauss en 1967 marque la naissance de ce qu'on appelle l'École de Constance. Cette étude ne sera traduite en français que dix ans plus tard (Paris, Gallimard, 1978). 
contexte socio-culturel dans lequel s'inscrit le texte (allusions littéraires à d'autres textes et références au contexte historique et social de l'œuvre en question). Selon Yves Gilli, le répertoire textuel, par sélection et répétition de schémas connus, "établit un horizon qui tient lieu de cadre entre le texte et le lecteur" 2 . Le texte attire ainsi l'attention du lecteur sur des systèmes signifiants qui lui sont familiers.

Iser analyse les phénomènes d'ordre psychologique qui se déclenchent chez le lecteur au moment de sa lecture. Son activité de synthèse, au tout début, se situe au niveau de l'inconscient: le lecteur construit des "synthèses passives" 3 . Cela explique pourquoi le lecteur est pris et s'implique totalement pendant sa lecture ${ }^{4}$. Au moyen d'images inconscientes, le lecteur se représente ce que le texte lui donne à lire. Mieux, il construit sa propre image synthétique du texte, en essayant de donner une cohérence à un maximum de données fournies par celui-ci. Ces synthèses passives sont suivies de synthèses de prédicats, cette fois-ci conscientes. À l'aide du langage, le lecteur construit une synthèse du texte.

D'après Iser, la lecture, comme le texte, sont des événements en cours 5 . Pendant la lecture, le lecteur a un point de vue mobile ${ }^{6}$. Il vit entre l'attente et le souvenir. Son activité débouche sur la création de synthèses, où il supprime toutes les tensions, les contradictions ou les manques d'information issus du texte luimême. Ces synthèses ne sont jamais définitives, puisque pendant la lecture le lecteur est constamment exposé à de nouvelles stratégies textuelles et à de nouveaux éléments de répertoire qui lui font modifier la synthèse de sa lecture.

L'activité du lecteur est stimulée par l'indétermination du texte, redevable de la présence de ce qu'Iser appelle "blancs" et

2 Yves Gilli, *Le texte et sa lecture. Une analyse de l'acte de lire selon W. Iser *, Semen 1. Lecture et lecteur, Paris, Les Belles Lettres, 1983, p. 109.

3 W. Iser, L'acte de lecture. Théorie de l'effet esthétique, Bruxelles, Mardaga, 1986, p. 245-286.

$4 \quad$ Ibid., p. 233.

5 Roman Ingarden a été le premier à signaler que le texte se caractérise par son indétermination. C'est cette indétermination, le fait qu'un texte ne soit jamais totalement construit, qui favorise l'interaction entre le texte et le lecteur (Das literarische Kunstwerk, Tübingen, Niemeyer, 1960).

6 Iser, op. cit., p. 199 et s. 
"négations"7. Partout où une suite de segments textuels est interrompue ou, au contraire, des segments opposés sont rapprochés, nous nous trouvons face à un blanc qui stimule l'activité du lecteur. Ces blancs, ou trous, ont pour but de signaler le besoin d'établir des relations entre des segments et de réorganiser le point de vue de la lecture.

Dans Volkswagen Blues, à cause des indéterminations du texte, deux constructions différentes sont mises en parallèle lors de notre lecture, l'une positive, l'autre négative. Il y a, d'une part, celle de Jack, qui veut à tout prix interpréter tous les signes par référence à un Théo héroïque; d'autre part, il y a des stratégies textuelles qui tendent à défaire cette synthèse par l'emploi d'éléments de répertoire, d'indéterminations et de mises en abyme.

Les premières pages de Volkswagen Blues sont importantes parce qu'elles posent un problème de lecture qui va conditionner tout le récit. Non sans raison, Jack avoue plus tard que la première phrase doit entraîner le lecteur dans sa lecture. Dès le début, l'attention du lecteur est attirée sur une carte postale mystérieuse qui renvoie à un certain Théo, frère de Jack. Il s'agit d'une carte écrite en ancien français, qui, de plus, reproduit une écriture qui n'est pas celle du destinateur.

Deux personnalités se trouvent à l'origine du texte. Ce dédoublement de destinateurs est repris au niveau des destinataires. En effet, notre lecture de la carte photocopiée coïncide avec celle de Jack, qui a sous les yeux la carte originale. C'est ainsi que nous commençons à identifier nos synthèses de lecture à celles de $\mathrm{Jack}^{8}$. Comme réaction à cette carte mystérieuse le texte propose deux synthèses possibles:

- C'est une sorte de message qu'il vous envoyait, vous ne pensez pas?

- À moins que ce soit une blague. (p. 15)

Voilà deux hypothèses auxquelles, de prime abord, on pourrait accorder le même degré de crédibilité. Notre lecture étant influencée par celle de Jack, nous sommes portés à adhérer à la première hypothèse. Il n'empêche que les stratégies textuelles ont déjà annoncé

Ibid., p. 318 et 365 .

8 Comme le signale Bertrand Gervais, l'identification du lecteur au héros est un mécanisme d'adhésion à l'univers narratif (Récits et actions. Pour une théorie de la lecture, Longueuil, Le Préambule, 1990, p. 321). 
une autre hypothèse de lecture: la possibilité d'une blague. Celle-ci sera l'horizon de notre lecture, le thème étant le fait que Théo a transmis un message lié au contexte de l'exploration américaine et qu'il s'est identifié en quelque sorte à Jacques Cartier.

Or, on constate que la carte postale, porteuse d'un message, est le point de départ d'une recherche non pas du sens (de son message possible) mais du destinateur. Un destinateur que Jack va identifier à un héros de la conquête américaine. La lecture de la carte de Théo marque le début d'un long voyage ${ }^{9}$. Jack bâtit notre code de lecture, soit un système d'attentes liées à un répertoire de livres et de récits de voyages américains. Voulant en savoir davantage à propos de ce qui entoure Théo, il se nourrit de toute une série de récits. Une lecture particulière de la carte entraîne donc d'autres lectures et relectures.

La lecture que fait Jack de La pénétration du continent américain nous renseigne sur deux choses. Elle nous permet de savoir que Saint Louis était un point de départ de toutes sortes d'expéditions, où les Canadiens étaient "les mieux faits pour supporter en leur qualité de pagayeurs, les rigueurs d'une expédition en canot. (p. 45). Elle nous fait aussi comprendre le parallèle qu'établit Jack entre son frère et les anciens pionniers. Une phrase nous aide à identifier ce système d'interprétation de Jack: "L'homme ne peut s'empêcher de sourire" (p. 46). Le corps de Jack répond aux stimuli externes: "Il ferma le livre, croisa les mains derrière sa tête et le chat vint se coucher sur son ventre" (p. 46). Les gestes dévoilent sa rêverie. À travers ce monde de signes gestuels nous prenons conscience de ce qui se passe dans la tête de Jack, nous faisons notre propre construction du roman, et croyons qu'elle coïncide avec la sienne. La joie de Jack est le signe de cette identification des exploits des anciens à ceux de son frère aîné ${ }^{10}$. Différentes représentations de l'acte de lecture

9 En ce sens, le roman représente clairement ce que Gilli a déjà remarqué: la lecture entraîne non seulement des effets esthétiques, mais aussi des actions.

10 Dans ces premiers moments de notre lecture, nous effectuons ce que F. Rutten appelle un "colmatage textuel*, une *opération de production de sens complémentaire aux opérations de production de sens qui sont déclenchées, de façon directe ou indirecte, par la perception de l'artefact. Le colmatage textuel supplée à une insuffisance de l'artefact pris comme source d'instruction. "Par artefact il faut entendre *le texte dans sa matérialité. Voir F. Rutten, *Sur les notions de texte et de 
56

s'unissent pour établir un système cohérent d'interprétation, et créer un code de lecture: Théo = nouveau héros contemporain.

Mais voilà que les possibilités laissées de côté prennent le dessus pour introduire un point de vue nouveau qui nous oblige à changer le nôtre. En effet, peu après, Jack s'intéresse à Étienne Brûlé et demande des renseignements au Pinkerton de la bibliothèque de Toronto. Brûlé est devenu une sorte d'obsession pour lui. Il pose des questions avec "agressivité", "tristesse" et "lassitude". Trois substantifs qui s'opposent radicalement au champ sémantique dominant dans les chapitres précédents, où on parlait d'“espoir" (p. 45), de "sourire", de "paroles hésitantes" (p. 26). Nous sommes ainsi frappés par un brusque changement d'attitude de Jack, à tel point que toute une série de questions et de réponses commencent à nous envahir. Le sourire initial se change en malaise dès qu'il entend dire que Brûlé était un bum. Ainsi, la visite à la bibliothèque de Toronto, unie à la lecture de Toronto during the French Regime, sert de charnière entre la première synthèse de lecture et une nouvelle lecture du roman qui n'est plus univoque: Théo $=b u m$; Théo $=$ héros. Une nouvelle voie a été ouverte, qui crée du suspense et oblige Jack, ainsi que nous-mêmes, à osciller d'un axe à l'autre selon les circonstances. À cause de l'indétermination du texte, ni notre construction ni celle de Jack ne peuvent être définitives. Jack croyait avoir affaire à un héros, et il apprend que celui-ci a de fortes chances d'être un simple bum, un inadapté social, un clochard.

Dans Volkswagen Blues, la synthèse établie jusqu'à présent est brisée par un mot qui introduit deux types de blancs. Premièrement, est-ce que Brûlé était vraiment un bum? Le Pinkerton aurait-il raison? Deuxièmement, pourquoi Jack tombe-t-il soudainement en dépression? Est-ce simplement parce qu'il identifie Brûlé et Théo? Si oui, pourquoi veut-il conserver une image héroïque de son frère? Parce qu'il l'aime? Comme lecteurs, notre attente est éveillée de telle sorte que nous cherchons à faire disparaitre ces lacunes le plus tôt possible. C'est peut-être pour cela que nous adhérons assez vite aux explications psychologiques que donnent les personnages ${ }^{11}$. Cela nous semble logique

lecture dans une théorie de la réception *, Revue des sciences bumaines, vol. 1, no 177 , p. 80.

11 Le contenu psychologique est fondamental dans la lecture comme construction (Tzvetan Todorov, * La lecture comme construction *, Les genres du discours, Paris, Seuil, 1978, p.43). 
de les entendre discuter, chercher une raison à la dépression de Jack. Au fond, cela nous soulage, car cela facilite notre tâche de créer un nouvel ordre dans notre lecture.

Ainsi, un peu plus tard, la Sauterelle et Jack dévoilent leurs propres interprétations des événements en cours: la Sauterelle croit que Jack veut défendre Brûlé parce qu'inconsciemment il croit que Théo a fait quelque chose d'illicite. Jack, pour sa part, parle d'un sentiment de culpabilité. Il croit que la carte était un SOS qu'il a manqué. De cette façon, à travers le dialogue des personnages, on introduit du nouveau, soit des éléments psychologiques: la culpabilité et le refoulement. Ceci nous rappelle encore cette piste lancée au début du roman: et si ce n'était qu'une blague? Notre mémoire se met en marche et commence à contredire la synthèse que Jack et la Sauterelle essaient de nous faire adopter.

Le narrateur intervient pour analyser le cas de Jack: "dans le plus secret de son couur, c'était comme si tous les héros du passé étaient encore des héros" (p. 79). Après une telle explication, nous pouvons nous sentir un peu "vexés" dans notre orgueil de lecteurs/décodeurs et penser que le narrateur en a trop dit. Or, ce n'est que lors d'une relecture ("qui n'a pas relu n'a pas lu ", dit la Sauterelle) que nous nous apercevons que le narrateur non seulement n'en a pas trop dévoilé, mais qu'il a réussi à nous tromper (tel est pris qui croyait prendre!). En effet, le narrateur dit tout simplement ce que Jack croit être réel. D'une part, ce "comme si " suivi d'un imparfait témoigne du caractère hypothétique et presque impossible de la comparaison. D'autre part, le narrateur ne dit mot sur l'identité de ces héros. Qui est le héros de Jack? Tout simplement son frère? Cela paraît un peu trop soudain, étant donné qu'il a passé quinze ans sans même lui écrire un mot. Voilà un blanc qui reste vide pendant une grande partie du roman et qui ne sera rempli qu'à la fin. Pour nous, lecteurs, le premier volet du roman finit avec cette quête indéterminée. Qui cherchet-on vraiment?

Le roman joue sur deux paires de dichotomies: bum/héros et réalité/rêve. Dans Volkswagen Blues, la rêverie est toujours déclenchée par des circonstances qui entourent Jack. À ce propos, on peut mentionner la scène de réconciliation de la Grande Sauterelle avec elle-même. On voit la Sauterelle dormir au cimetière rêvant à sa mère indienne. Cet épisode, qui semble laisser de côté Jack pour focaliser l'attention sur la Sauterelle, apporte 
cependant quelques éléments qui renforcent les attentes qui s'étaient créées chez le lecteur. En effet, Jack souhaite bonne nuit aux différents esprits des morts, s'allonge tout habillé sur le lit et avoue de façon indirecte que les rêves ont une consistance propre. Contrairement à la Sauterelle, qui croit que cette nuit-là uil ne s'est rien passé du tout", Jack demande si elle a rêvé à quelque chose et, à la réponse affirmative, il réplique "alors, vous ne pouvez pas dire qu'il ne s'est rien passé du tout...Vous voyez bien" (p. 88). Jack se présente comme un personnage du rêve. Dans les autres chapitres, on en avait sans doute eu l'impression. Cette thématique avait toutefois été obscurcie par la quête du frère et par les allusions aux différentes lectures de Jack. Maintenant, le rêve prend le dessus et devient le thème du roman.

Tout un système de redondances se crée autour de l'axe de la rêverie. En effet, une chanson, les mots d'un prix Nobel et ceux d'un vieillard permettent de mieux comprendre sa portée. D'une part, la chanson qui contient le mot "Amérique" concrétise un aspect fondamental dans la rêverie de Jack. La présence de ce mot met bien en relief le caractère rêveur du jeune homme, qui est capable de rêver rien qu'à l'écoute d'un groupe de sonorités. Pour lui, entendre le mot "Amérique " entraîne tout de suite une réaction dans son corps. Il sent "bouger quelque chose au milieu des brumes qui obscurcissaient son cerveau "(p. 100). La chanson est associée au bonheur qui "vient toujours avec la peine". "Les voyages forment la jeunesse", entend-il. La chanson rallume son espoir. Par le pouvoir évocateur des mots, l'Amérique acquiert un visage positif qui amortit les soupçons et les craintes des chapitres antérieurs.

Un peu plus loin, les mots de l'écrivain Saul Bellow dans ses Aventures d'Augie March renforcent cette attitude rêveuse de Jack. Dès le début, ce roman parle d'une Amérique conçue comme la terre des retrouvailles. On parle d'une "sorte de Colomb, capable de rejoindre tous ceux qui sont à portée de la main" (p. 111). Quoi de mieux pour renforcer la rêverie et les espoirs cachés de Jack?

Enfin, une troisième scène nous éclaire sur la nature de sa rêverie. À la vue d'un vieillard qui regarde le fleuve, Jack est capable de concrétiser l'image de son propre rêve. En fait, c'est un rêve d'éternité, d'immortalité. De cette manière, on constate que ces chapitres ne sont qu'une autre manière d'aborder le sujet principal: les attentes intimes de Jack, sa quête, ses rêveries 
profondes. Ces chapitres à caractère fortement lyrique marquent une pause dans le rythme de lecture et accordent au lecteur le temps nécessaire pour remodeler l'image du roman.

Après ces épisodes, un autre moment clé du roman se présente: la lecture d'un article sur Théo dans le journal d'Independance, où Thêo est accusé de vol. Il n'y a pas de doute là-dessus. Il s'agit d'un texte à caractère référentiel ${ }^{12}$. Or, si toutes les indéterminations du roman semblent pouvoir finir ici, il n'en sera rien. Jack, conscient comme nous que le moment est arrivé de savoir si Théo était ou non un hors-la-loi, provoque les événements afin que la vérité soit escamotée.

En effet, au lieu de profiter des vingt dernières minutes avant la fermeture des bureaux pour fouiller dans les jugements de la cour et savoir si 'Théo avait été condamné, il trouve que le temps presse et qu'il faut partir ${ }^{13}$. Voilà le blanc fondamental du roman ${ }^{14}$. Notre imaginaire est ainsi activé ${ }^{15}$. Les silences du texte sont un stimulus dans son activité de construction.

Dès que Jack et la Sauterelle entament la piste de l'Oregon, ils sont accompagnés par The Oregon Trail Revisited.

Elle ferma la radio et demanda à Jack comment les choses se passaient dans son livre.

- Pas trop mal, dit-il, à part la poussière...

- Les émigrants avalent de la poussière?

- Ils n'ont jamais mangé autant de poussière de leur vie. [...]

les chariots avancent en lignes parallèles [...]

12 Dans ce genre de textes il n'y a pas de disjonction; on cherche *à obtenir du destinataire l'acceptation d'une intention relative à un état de faits . (Iser, op.cit., p. 322).

13 Notons au passage le parallélisme existant entre cette scène et un épisode fondamental dans la construction du Vieux chagrin (Leméac/Actes sud, 1989). Là, le protagoniste (Jim), voyant hésiter Bungalow, refuse de lui poser des questions à propos de Marika. Lc lecteur ne saura jamais si Marika existe, de la même façon qu'il ne saura jamais ce qui s'est passé avec Théo.

14 Ce sont ces blancs qui mettent en question la slisibilité " du roman (au sens d'univocité de la signification). Voir à ce propos Philippe Hamon, - Un discours contraint *, Poétique, n' 16, 1973, p. 422 et s.

15 Comme le signale Charles Grivel, * tout délai m'attache à la fin. Toute fin m'arrache et toute fin m'exclut. ("Monomanie de la lecture (notes éparses) *, Rèue des sciences bumaines, vol. 1, n 177, janvier-mars 1980 , p. 99). 
60

- Vous n'avez pas vu de cours d'eau?

- Il y avait une sorte d'étang, dit Jack, mais l'eau était empoisonnée.

- Qui vous a dit ça? Vous avez vu une affiche avec une tête de mort?

- Mais non!

Jack secoua la tête en riant.

- Mais non, dit-il. Pas besoin d'affiche. Il suffisait de sentir cette odeur de pourriture... Le guide a dit que si on se risquait à boire, c'était la dysenterie [...]

- Comme ça vous avez un guide? [...]

- Bien sûr, dit Jack.

- Votre expédition est très bien organisée! [...]

- On a choisi un francophone pour qu'il soit capable de s'entendre avec les Indiens.

-Un francophone... Frémont?... Bonneville?

- C'est un guide spécial, dit l'homme.

-Ah bon!... Il ne s'appellerait pas Théo, par hasard? (p. 176177)

La lecture de cet extrait est marquée par l'indétermination introduite par le pronom indéfini "on". Nous, lecteurs, avons l'impression que Jack est en train de parler avec la Sauterelle du manque d'eau qui affectait les émigrants. Or, à travers ce "on", et à partir de la question "Vous n'avez pas vu de cours d'eau?", nous ne savons plus' s'ils parlent de l'histoire écrite ou de leur histoire à eux. Cette indétermination est finalement levée au moment où Théo apparât comme guide de l'expédition. Ce n'est ni Kit Carson, ni Jim Bridger. C'est un francophone. De plus ce guide a, d'après Jack, un wagon master dont la "tete est pleine de brume" (p. 178). La correspondance entre la fiction et la réalité est d'ores et déjà parfaitement établie. La seule allusion à la brume suffit pour que le lecteur reconnaisse Jack derrière ce wagon master. La confusion entre le passé et le présent culmine avec l'emploi du pronom "nous" qui sans équivoque inclut Jack dans le récit:

De temps en temps, le guide va voir comment les choses se passent à l'arrière du convoi: c'est là que se trouvent les vaches et les chevaux que nous emmenons en Oregon, dans la vallée de la Willamette, où nous allons cultiver la terre et élever les animaux. (p. 179) 
Nous pourrions penser qu'il lit, mais le narrateur signale que "Jack s'interrompt un moment. Il n'était pas habitué à tant parler" (p. 179). Cela est confirmé par le fait que par la suite il parle à la troisième personne du pluriel, au moment où il reprend sa lecture en bonne et due forme.

Dans ce passage, nous semble-t-il, les véritables motivations du voyage de Jack se laissent entrevoir. Il ne cherche pas son frère-héros, il veut lui-même devenir un héros. Ce qu'il veut c'est faire revivre "la partie de moi qui a oublié de vivre" (p. 137). Théo ne serait qu'un prétexte, qu'un miroir où il voudrait voir sa propre image embellie. De là, ses dépressions. De là, son intérêt à conserver des images attirantes des héros. Le lecteur commence à pouvoir remplir les blancs du texte. C'est pour cela que le narrateur prend soin de créer tout un code linguistique visant à exalter The Oregon Trail Revisited: "leur livre préféré" (p. 175), "leur livre" (p. 206), "leur livre bien aimé" (p. 219).

S'il en est ainsi, c'est que ce livre est source de rêverie et permet à Jack d'échapper à la réalité ${ }^{16}$. L'axe rêve/réalité est renforcé par la suite lorsque Jack et la Sauterelle rencontrent un "voyageur" qui, sans l'avouer, se prend pour Hemingway. En fait, c'est le double de Jack qui, lui, se prend pour un colon du XIX siècle. Nous constatons donc que, par de petits détails d'ordre grammatical, les stratégies textuelles provoquent un écart entre nos synthèses de lecture et celles que le protagoniste veut faire passer. Par ce jeu de pronoms, nous voyons que Jack ne cherche pas son frère, comme il le prétend, mais sa propre image.

À la fin du roman, nous constatons encore que la présence d'un document à caractère non littéraire, une photo contenue à l'intérieur de Beat Angels, déclenche des événements fondamentaux du récit. Curieusement, derrière la photo, on désigne Théo comme un "unidentified man". Cette inscription préfigure en l'inversant le dénouement de Volkswagen Blues, où l'on verra Théo dire à Jack "I don't know you ".

À la vue de Théo, l'hypothèse qui avait été laissée dans l'horizon de notre lecture s'impose comme synthèse valable. Ce "À moins que ce ne soit une blague" nous revient en tête et nous fait comprendre qu'en effet, l'identification de Théo avec un

16 La lecture devient ici un acte performatif. Lire, c'est faire, au sens fort du terme. Ce motif renvoie à Cervantès et à Don Quichotte. 
62

héros quelconque était dérisoire. En lisant cette carte où Jack avait identifié son frère aux héros du passé, Jack avait inauguré en même temps son propre cheminement d' héroïsation ". Mais il n'avait pas tenu compte de ce que, quinze ans s'étant écoulés, Théo n'était plus un jeune homme. Son étonnement ultime aurait pu être évité s'il n'avait pas négligé cette deuxième hypothèse proposée par le récit: ce n'est qu'une blague.

Mais notre synthèse ne s'arrête pas là. La fin du roman, en apparence décevante, signale que Jack sourit "malgré tout" (p. 290). Le dernier paragraphe, au lieu de clore le texte dans une configuration classique (Jack est déçu parce que Théo n'est pas un héros), stimule à la toute dernière minute notre imagination. Pourquoi sourit-il? N'a-t-il pas perdu des mois de voyage? Malgré tout, c'est le rêve qui le sauve. Il l'a accompagné pendant tout son voyage et, à la fin, il empêche l'aventure de tourner au drame.

En définitive, contrairement aux apparences, Volkswagen Blues n'est ni le roman de la quête impossible ni celui des héros déchus. Cela n'est qu'une partie du répertoire activé par le texte, qui empêche parfois de voir l'horizon sur lequel se construit la lecture du roman. Avec Poulin, on peut toujours "faire de beaux rêves "... 